\title{
RABEP1/PDGFRB Fusion Protein
}

National Cancer Institute

\section{Source}

National Cancer Institute. RABEP1/PDGFRB Fusion Protein. NCI Thesaurus. Code C99592.

A fusion protein (1318, 150 kDa) encoded by the RABEP1/PDGFRB fusion gene. This protein is comprised of $85 \%$ of the Rab GT Pase-binding effector protein 1, including 3 and a half of the coiled coil domains, fused to the C-terminal transmembrane and cytoplasmic domains of the beta-type platelet-derived growth factor receptor protein, including the protein kinase domain. 